\title{
Solution of the tumor-immune system by differential trans- form method
}

\author{
Mohamed Abd El Hady Kassem, A. A. Hemeda, M. A. Abdeen* \\ Department of Mathematics, Faculty of Science, Tanta University, Egypt.
}

\begin{abstract}
In this paper, differential transform method (DTM) is presented to solve Tumor-immune system at two initial conditions where two different cases of the interaction between tumor cells and effector cells. The system is presented to show the ability of the method for non-linear systems of differential equations. By using small iteration, the results of DTM are near the results of Runge-Kutta fourth-fifth order method (ode45 solver in MATLAB) and better than the results of Runge-Kutta second-third order method (ode23 solver in MATLAB). Also, the residual error of DTM's solutions approach zero. Therefore, DTM's solutions approximate exact solutions. Finally, we conclude formulae that we can find DTM's solutions, better than the results of RungeKutta second-third order method, in any interval we need.
\end{abstract}

Keywords: Kuznetsov and Taylor's model, differential transform method, Runge-Kutta fourth-fifth order method, Runge-Kutta second-third order method, tumor-immune system.

2010 MSC: 37N30.

(C)2020 All rights reserved.

\section{Introduction}

The immune system response consists of two different interacting responses: the cellular response ( $T$ lymphocytes) and the humoral response (B lymphocytes). The immune system identifies any stranger cells or tumor cells and destroys them. The immune response (in vivo) starts when tumor cells are recognized as being nonself. Then tumor cells are caught and absorbed by macrophages which can be found in all tissues in the body and circulate in the blood stream. Macrophages release series of cytokines which activate $\mathrm{T}$ helper cells which send signals to natural killers (NKs). T cells begin to multiply and release other cytokines that stimulate more T cells, B cells and NK cells. As the number of B cells increases, $\mathrm{T}$ helper cells send a signal to start the process of the production of antibodies. Antibodies circulate in the blood and attach to tumor cells, which implies that they are more quickly engulfed by macrophages or killed by natural killer cells.

\footnotetext{
${ }^{*}$ Corresponding author

Email addresses: mohd60_371@hotmail.com (Mohamed Abd El Hady Kassem), aahemeda@yahoo.com (A. A. Hemeda), mohammed3185@yahoo.com (M. A. Abdeen)
}

doi: $10.22436 /$ jnsa.013.01.02 
There are many mathematical models for describing interactions between tumor cells and immune cells as DeLisi and Rescigno's model [9], Kuznetsov and Taylor's model [16], Adam's model [1] and Magda's model [10]. These models are defining an ordinary differential equation (ODE) model for two main populations: effector cells and tumor cells. Also, there are many mathematical models for effective of immunotherapy on interactions between tumor cells and immune cells as Kirschner and Panetta's model [15] and the simplified model [21]. These models are defining an ordinary differential equation (ODE) model for three main populations: effector cells, tumor cells, and the concentration of Interleukin-2 (the main cytokine responsible for lymphocyte activation, growth and differentiation).

There are many methods to solve system of ordinary differential equations as fractional sumudu transform [14], Adomian decomposition method [5, 6], and differential transform method [17]. Differential transform method is a good numerical method to solve ordinary differential equation because it has high accuracy with minor error. Also, differential transform method is applied to nonlinear systems of ordinary differential equations $[8,17,18,20]$, fractional order nonlinear boundary value problems [11], higher-order nonlinear differential equations which have convolution terms [13] and some boundary value problems with convolution terms [12].

We present Kuznetsov and Taylor's model and show the phase space of this model. There are four equilibria are showed in the phase space of this model. Two equilibria are stable and other equilibria unstable. Two stable equilibria represent two different cases of interactions between cancer and the immune system in animal body (in-vivo). First, tumor dormant, the immune system can restrain cancer. Second, tumor escape case, the immune system cannot control of the increase of cancer which grow without any impediments. We solve Kuznetsov and Taylor's model by (DTM) (approximations to the solutions with in five terms) in two different cases; tumor dormant case and tumor escape case to examine the effectiveness of DTM method for solving Non-linear system of ordinary differential equations at different initial conditions. Also, we compare DTM's solution with Runge-Kutta fourth-fifth order method (ode45 solver in MATLAB) as exact solution and Runge-Kutta second-third order method (ode23 solver in MATLAB) in small interval after start of initial conditions, i.e., $[0,0.1]$. Also, we conclude the residual functions and we compute residual errors in two different cases, tumor dormant and tumor escape, for DTM's solutions. See $[3,4]$. Finally, by using maple, we conclude the two formulae for $x(a, b, t)$ (effector cells) and $y(a, b, t)$ (tumor cells) as function of initial conditions $\left(a=x_{0}\right.$ and $\left.b=y_{0}\right)$ and time $(t)$. Then, by using MATLAB, we use DTM procedure in small interval and repeat DTM procedure with change initial conditions to new initial conditions, to any time we need, until stability of solution (the suitable interval of solution). Also, in two cases, we compare DTM's solution with Runge-Kutta fourth-fifth order method (RK45) as exact solution and Runge-Kutta second-third order method (RK23) in the suitable interval of solution.

\section{Differential transform method}

The standard form of system of $\mathrm{N}$ ordinary differential equations of the $n$ order with conditions is considered, see $[7,17]$

$$
\begin{gathered}
\phi_{1}\left(x, y_{1}(x), y_{1}^{\prime}(x), \ldots, y_{1}^{(n)}(x), y_{2}(x), y_{2}^{\prime}(x), \ldots, y_{2}^{(n)}(x), \ldots, y_{N}(x), y_{N}^{\prime}(x), \ldots, y_{N}^{(n)}(x)\right)=0 \\
\phi_{2}\left(x, y_{1}(x), y_{1}^{\prime}(x), \ldots, y_{1}^{(n)}(x), y_{2}(x), y_{2}^{\prime}(x), \ldots, y_{2}^{(n)}(x), \ldots, y_{N}(x), y_{N}^{\prime}(x), \ldots, y_{N}^{(n)}(x)\right)=0 \\
\vdots \\
\phi_{N}\left(x, y_{1}(x), y_{1}^{\prime}(x), \ldots, y_{1}^{(n)}(x), y_{2}(x), y_{2}^{\prime}(x), \ldots, y_{2}^{(n)}(x), \ldots, y_{N}(x), y_{N}^{\prime}(x), \ldots, y_{N}^{(n)}(x)\right)=0,
\end{gathered}
$$

with initial values given by

$$
\begin{aligned}
& y_{1}\left(x_{0}\right)=y_{1_{0}}, y_{1}^{\prime}\left(x_{0}\right)=y_{1_{0}}^{\prime}, \ldots, y_{1}^{n-1}\left(x_{0}\right)=y_{1_{0}}^{n-1}, \\
& y_{2}\left(x_{0}\right)=y_{2_{0}}, y_{2}^{\prime}\left(x_{0}\right)=y_{2_{0}}^{\prime}, \ldots, y_{2}^{n-1}\left(x_{0}\right)=y_{2_{0}}^{n-1},
\end{aligned}
$$




$$
y_{N}\left(x_{0}\right)=y_{N_{0}}, y_{N}^{\prime}\left(x_{0}\right)=y_{N_{0}}^{\prime}, \ldots, y_{N}^{n-1}\left(x_{0}\right)=y_{N_{0}}^{n-1},
$$

where $\phi_{i}$ for $i=1(1) N$ are nonlinear continuous functions of their arguments. The transformation of the $k^{\text {th }}$ derivative of a function in one variable is as follows:

$$
Y(k)=\frac{1}{k !}\left[\frac{d^{k} y}{d x^{k}}(x)\right]_{x=x_{0}},
$$

and the inverse transformation is defined by

$$
y(x)=\sum_{k=0}^{\infty} Y(k)\left(x-x_{0}\right)^{k}
$$

see [22]. The following theorems can be deduced from equations (2.1) and (2.2).

Theorem 2.1. If $y(x)=y_{1}(x) \pm y_{2}(x)$, then $Y(k)=Y_{1}(k) \pm Y_{2}(k)$.

Theorem 2.2. If $\mathrm{y}(\mathrm{x})=\mathrm{cy}_{1}(\mathrm{x})$, then $\mathrm{Y}(\mathrm{k})=\mathrm{cY} \mathrm{Y}_{1}(\mathrm{k})$ where $\mathrm{c}$ is a constant.

Theorem 2.3. If $y(x)=\frac{d^{n} y_{1}(x)}{d x^{n}}$, then $Y(k)=\frac{(k+n) !}{k !} Y_{1}(k+n)$.

Theorem 2.4. If $y(x)=y_{1}(x) y_{2}(x)$, then $Y(k)=\sum_{k_{1}=0}^{k} Y_{1}\left(k_{1}\right) Y_{2}\left(k-k_{1}\right)$.

Theorem 2.5. If $\mathrm{y}(\mathrm{x})=\mathrm{x}^{\mathrm{n}}$, then $\mathrm{Y}(\mathrm{k})=\delta(\mathrm{k}-\mathrm{n})$ where $\delta(\mathrm{k}-\mathrm{n})= \begin{cases}1, & \mathrm{k}=\mathrm{n}, \\ 0, & \mathrm{k} \neq \mathrm{n} .\end{cases}$

Theorem 2.6. If $y(x)=y_{1}(x) y_{2}(x) \cdots y_{n}(x)$, then

$$
Y(k)=\sum_{k_{n-1}=0}^{k} \sum_{k_{n-2}=0}^{k_{n-1}} \cdots \sum_{k_{2}=0}^{k_{3}} \sum_{k_{1}=0}^{k_{2}} Y_{1}\left(k_{1}\right) Y_{2}\left(k_{2}-k_{1}\right) \cdots Y_{n-1}\left(k_{n-1}-k_{n-2}\right) Y_{n}\left(k-k_{n-1}\right) .
$$

Theorem 2.7. If $\mathrm{y}(\mathrm{x})=\mathrm{e}^{\lambda x}$, then $\mathrm{Y}(\mathrm{k})=\frac{\lambda^{\mathrm{k}}}{\mathrm{k} !}$, where $\lambda$ is a constant.

Theorem 2.8. If $y(x)=\sin (\omega x+\alpha)$, then $Y(k)=\frac{\omega^{k}}{k !} \sin \left(\frac{k \pi}{2}+\alpha\right)$, where $\omega$ and $\alpha$ are constants.

Theorem 2.9. If $y(x)=\cos (\omega x+\alpha)$, then $Y(k)=\frac{\omega^{k}}{k !} \cos \left(\frac{k \pi}{2}+\alpha\right)$, where $\omega$ and $\alpha$ are constants. [19].

The proofs of Theorems 2.1-2.6 are available in [2] and the proofs of Theorems 2.7-2.9 are available in

\section{Kuznetsov and Taylor's model}

The nonlinear system describes the interaction between tumor cells and effector cells in-vivo (inside animal body), see [16], is

$$
\frac{d x}{d t}=\sigma+\frac{\rho x y}{\eta+y}-\mu x y-\delta x, \quad \frac{d y}{d t}=r_{2} y(1-\beta y)-x y,
$$

where $x$ denotes the dimensionless density of effector cells, $y$ stands for the dimensionless density of the population of tumor cells, $\sigma=0.1181$ (the parameter of normal production of effector cells), $\mu=0.00311$ (the parameter of effector cells are destroyed by tumor cells), $\delta=0.3743$ (the parameter of death of effector cells) , $r_{2}=1.636$ (the parameter of maximal growth rate of tumor cells), $\beta=0.002\left(\beta^{-1}\right.$ is the parameter of maximal carrying capacity of the biological environment for tumor cells), $\rho=1.131$ and $\eta=20.19$ (the term of Michaelis-Menten form characterizes the rate at which effector cells accumulate in the region of tumor cells due to the presence of the tumor). 


\subsection{Phase Space}

There are four equilibria according to the estimated values of parameters. The phase space is shown in Figure 1 (a). The four equilibria are indicated and denoted " $\mathrm{B}$ ", " $\mathrm{C}$ ", " $\mathrm{D}$ ", and " $\mathrm{A}$ " which is the free-tumor equilibrium. The variable $x$ is the non-dimensional effector cell population and $y$ the non-dimensional tumor cell population. The positive equilibria labeled " $\mathrm{B}$ " and " $\mathrm{D}$ " are both stable. In addition, the steady state " $\mathrm{B}$ " is characterized by a relatively little number of tumor cells and we call it the "dormant tumor" equilibria. However, the steady state " $\mathrm{D}$ ", characterized by a relatively high number of tumor cells and low number of effector cells, corresponds to relatively "uncontrolled" tumor growth or "tumor escape". One dimensional stable manifold of the steady state " $\mathrm{C}$ " partitions the basin of attraction for each of these attractors. Initial conditions beginning blow and the right of this separatrix (e.g., initial conditions (i) and (iii) in Figure 1 (b)) asymptotically approach the dormant tumor steady state "B". As for the initial conditions above the separatrix (e.g., initial conditions (ii) and (iv) in Figure 1 (b)), the tumor escape the steady state "D".

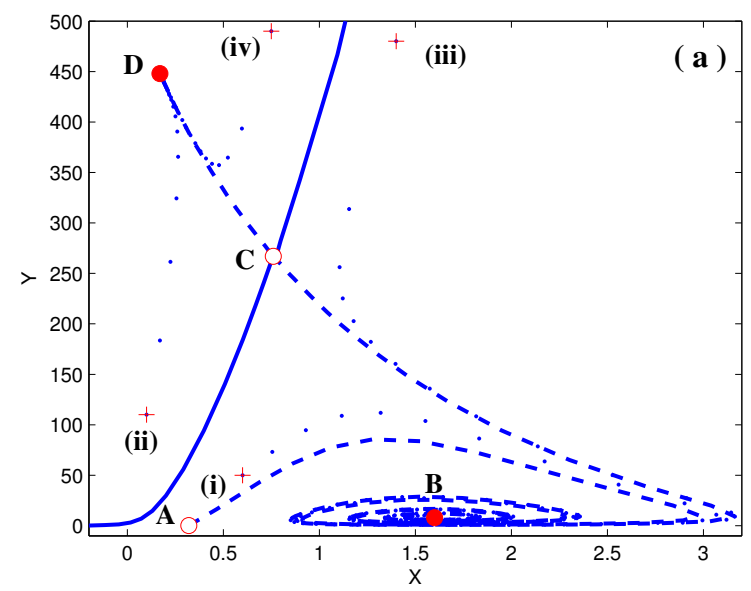

(a) Phase space.

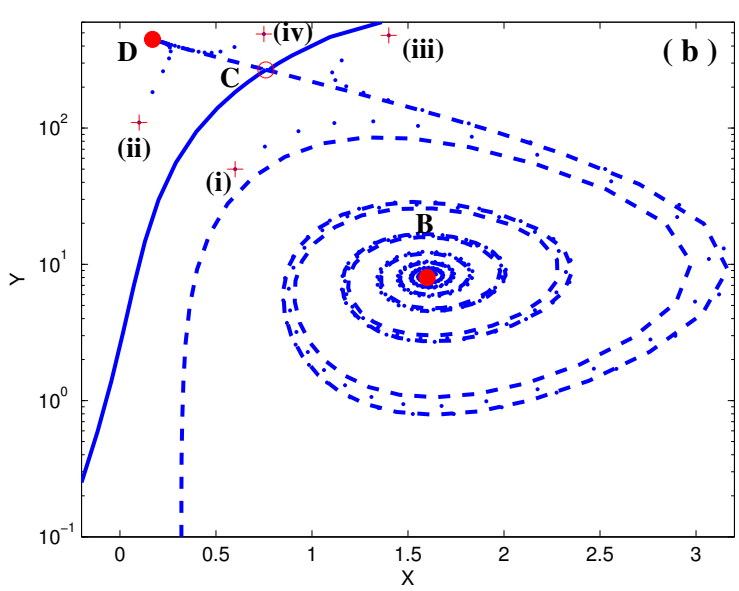

(b) Phase space with a logarithmic ordinate.

Figure 1: Stable steady states [•], saddle steady states [O], one-dimensional stable manifold of steady state " $\mathrm{C}$ " [ — ], onedimensional unstable manifolds of steady states " $\mathrm{A}$ " and "C" [ - - ] and initial conditions for transients [+].

\section{Numerical solutions at small interval after start of initial conditions}

We solve Kuznetsov and Taylor's system with two different initial values: $x(0)=1, y(0)=1$ (dormant tumor) and $x(0)=0.1, y(0)=25$ (tumor escape). We compare the solutions of the system; the effector cells $x(t), x_{R K 23}(t)$, and $x_{R K 45}(t)$ and tumor cells $y(t), y_{R K 23}(t)$, and $y_{R K 45}(t)$, by three numerical methods: DTM, the Runge-Kutta second-third order method (RK23), and the Runge-Kutta fourth-fifth order method (RK45) (as exact solution). We multiply $(\eta+y)$ in the first equation of system (3.1), we obtain

$$
\eta \frac{d x}{d t}+y \frac{d x}{d t}=\eta \sigma+\sigma y-\eta \delta x+(\rho-\delta-\eta \mu) x y-\mu x y^{2}, \quad \frac{d y}{d t}=r_{2} y(1-\beta y)-x y .
$$

By using the theorems in Section 2, the system (4.1) is transformed as

$$
\begin{aligned}
& \eta(k+1) X(k+1)+\sum_{r=0}^{k}(k+1-r) Y(r) X(k+1-r) \\
& \quad=\delta(k-0) \eta \sigma+\sigma Y(k)-\eta \delta X(k)+(\rho-\delta-\eta \mu) \sum_{r=0}^{k} X(r) Y(k-r)-\mu \sum_{r=0}^{k} X(k-r) \sum_{s=0}^{r} Y(s) Y(r-s),
\end{aligned}
$$




$$
(k+1) Y(k+1)=r_{2} Y(k)-r_{2} \beta \sum_{r=0}^{k} Y(r) Y(k-r)-\sum_{r=0}^{k} X(r) Y(k-r) .
$$

Simplifying the transformed system (4.2), we get

$$
\begin{aligned}
X(k+1)= & \frac{1}{(k+1)(\eta+Y(0))}\left[\delta(k-0) \eta \sigma+\sigma Y(k)-\eta \delta X(k)+(\rho-\delta-\eta \mu) \sum_{r=0}^{k} X(r) Y(k-r)\right. \\
& \left.-\mu \sum_{r=0}^{k} X(k-r) \sum_{s=0}^{r} Y(s) Y(r-s)-\sum_{r=1}^{k}(k+1-r) Y(r) X(k+1-r)\right], \\
Y(k+1)= & \frac{1}{k+1}\left[r_{2} Y(k)-r_{2} \beta \sum_{r=0}^{k} Y(r) Y(k-r)-\sum_{r=0}^{k} X(r) Y(k-r)\right] .
\end{aligned}
$$

\subsection{First, tumor dormant case}

By using MAPLE, we solve the system (4.3) at $X(0)=1$ and $Y(0)=1$, we find

$$
\begin{aligned}
& X(1)=-0.2059357668000 \text { ， } \\
& Y(1)=0.632728000000 \text {, } \\
& X(2)=0.0484702004100 \text {, } \\
& \mathrm{Y}(2) 7=0.302105101300 \text {, } \\
& X(3)=-0.0028213519070 \text {, } \\
& Y(3)=0.090227688740 \text {, } \\
& X(4)=0.0007562636005 \text {, } \\
& Y(4)=0.022477654190 \text {, } \\
& X(5)=0.0000477069303 \text {, } \\
& Y(5)=0.003688674208 \text {, }
\end{aligned}
$$

then

$$
\begin{aligned}
& x(t)=1-0.2059357668 t+0.04847020041 t^{2}-0.002821351907 t^{3}+0.0007562636005 t^{4}+0.0000477069303 t^{5}, \\
& y(t)=1+0.632728 t+0.3021051013 t^{2}+0.09022768874 t^{3}+0.02247765419 t^{4}+0.003688674208 t^{5} .
\end{aligned}
$$

By using MATLAB, Tables 1 and 2 show the results of the solution of Kuznetsov and Taylor's model at $x(0)=1$ and $y(0)=1$ present the absolute errors between the solutions $x_{R K 23}(t)$ and $y_{R K 23}(t)$ obtained by using RK23, the solutions $x(t)$ and $y(t)$ obtained by using DTM, and the solutions $x_{R K 45}(t)$ and $y_{R K 45}(t)$ obtained by using RK45. We get a good approximation by using presented method with only five iterations.

\subsection{Second, tumor escape case}

By using MAPLE, we solve the system (4.3) at $X(0)=0.1$ and $Y(0)=25$, we find

$$
\begin{array}{ll}
X(1)=0.135464152500, & Y(1)=36.3550000000, \\
X(2)=0.026433733440, & Y(2)=23.2534990900, \\
X(3)=0.010136089790, & Y(3)=7.3343009640, \\
X(4)=-0.004741179615, & Y(4)=0.0422513693, \\
X(5)=-0.002353362664, & Y(5)=-1.0628658550,
\end{array}
$$

then

$$
\begin{aligned}
& x(t)=0.1+0.1354641525 t+0.02643373344 t^{2}+0.01013608979 t^{3}-0.004741179615 t^{4}-0.002353362664 t^{5}, \\
& y(t)=25+36.355 t+23.25349909 t^{2}+7.334300964 t^{3}-0.0422513693 t^{4}-1.062865855 t^{5} .
\end{aligned}
$$

By using MATLAB, Tables 3 and 4 show the results of the solution of Kuznetsov and Taylor's model at $x(0)=0.1$ and $y(0)=25$ present the absolute errors between the solutions $x_{R K 23}(t)$ and $y_{R K 23}(t)$ obtained by using RK23, the solutions $x(t)$ and $y(t)$ obtained by using DTM, and the solutions $x_{R K 45}(t)$ and $y_{R K 45}(t)$ obtained by using RK45. We get a good approximation by using presented method with only five iterations. 
Table 1: The effector cells $x(t)$ obtained by DTM, the absolute error between $x_{R K 23}(t)$ and $x_{R K 45}(t)$, and the absolute error between $x(t)$ and $x_{R K 45}(t)$ at $x(0)=1$ and $y(0)=1$.

\begin{tabular}{|c|c|c|c|}
\hline$t$ & $x(t)$ with DTM & $\left|\chi_{R K 23}(t)-\chi_{R K 45}(t)\right|$ & $\left|x(t)-\chi_{R K 45}(t)\right|$ \\
\hline 0 & 1 & 0 & 0 \\
0.005 & 0.998971532568814 & $3.76920716860241 \mathrm{e}-013$ & $3.55493412484975 \mathrm{e}-013$ \\
0.010 & 0.997945486538256 & $3.87412324442948 \mathrm{e}-012$ & $7.10209668852713 \mathrm{e}-013$ \\
0.015 & 0.996921859809352 & $6.42674802264764 \mathrm{e}-012$ & $1.06470388061553 \mathrm{e}-012$ \\
0.020 & 0.995900650294504 & $9.95037385820297 \mathrm{e}-012$ & $1.41830991395864 \mathrm{e}-012$ \\
0.025 & 0.994881855917514 & $1.25265353645432 \mathrm{e}-011$ & $1.77102776888205 \mathrm{e}-012$ \\
0.030 & 0.993865474613600 & $1.60770285972944 \mathrm{e}-011$ & $2.12230233387345 \mathrm{e}-012$ \\
0.035 & 0.992851504329413 & $1.86770598986641 \mathrm{e}-011$ & $2.47168951972299 \mathrm{e}-012$ \\
0.040 & 0.991839943023054 & $2.22548646178211 \mathrm{e}-011$ & $2.81719092498633 \mathrm{e}-012$ \\
0.045 & 0.990830788664094 & $2.48793208257325 \mathrm{e}-011$ & $3.15791837124380 \mathrm{e}-012$ \\
0.050 & 0.989824039233593 & $2.84847700982027 \mathrm{e}-011$ & $3.48976403330425 \mathrm{e}-012$ \\
0.055 & 0.988819692724112 & $3.11342063241682 \mathrm{e}-011$ & $3.80973030900122 \mathrm{e}-012$ \\
0.060 & 0.987817747139737 & $3.47678552614639 \mathrm{e}-011$ & $4.11148892709434 \mathrm{e}-012$ \\
0.065 & 0.986818200496097 & $3.74427155946933 \mathrm{e}-011$ & $4.38882263864571 \mathrm{e}-012$ \\
0.070 & 0.985821050820375 & $4.11051193083267 \mathrm{e}-011$ & $4.63162841413123 \mathrm{e}-012$ \\
0.075 & 0.984826296151334 & $4.38057368157274 \mathrm{e}-011$ & $4.82891504560712 \mathrm{e}-012$ \\
0.080 & 0.983833934539331 & $4.74973393949085 \mathrm{e}-011$ & $4.96591656684586 \mathrm{e}-012$ \\
0.085 & 0.982843964046333 & $5.02240471433879 \mathrm{e}-011$ & $5.02597963247808 \mathrm{e}-012$ \\
0.090 & 0.981856382745940 & $5.39452926773265 \mathrm{e}-011$ & $4.98623364819650 \mathrm{e}-012$ \\
0.095 & 0.980871188723399 & $5.66987568006994 \mathrm{e}-011$ & $4.82314188587907 \mathrm{e}-012$ \\
0.100 & 0.979888380075622 & $5.71815927941088 \mathrm{e}-011$ & $4.50539605623135 \mathrm{e}-012$ \\
\hline
\end{tabular}

Table 2: The tumor cells $y(t)$ obtained by DTM, the absolute error between $y_{R K 23}(t)$ and $y_{R K 45}(t)$, and the absolute error between $y(t)$ and $y_{R K 45}(t)$ at $x(0)=1$ and $y(0)=1$.

\begin{tabular}{|c|c|c|c|}
\hline $\mathrm{t}$ & $\mathrm{y}(\mathrm{t})$ with DTM & $\left|\mathrm{y}_{R K 23}(\mathrm{t})-\mathrm{y}_{R K 45}(\mathrm{t})\right|$ & $\left|\mathrm{y}(\mathrm{t})-\mathrm{y}_{R K 45}(\mathrm{t})\right|$ \\
\hline 0 & 1 & 0 & 0 \\
0.005 & 1.00317120392005 & $4.72932804029824 \mathrm{e}-012$ & $3.10862446895044 \mathrm{e}-015$ \\
0.010 & 1.00635758096296 & $5.71416247652223 \mathrm{e}-011$ & $1.75415237890775 \mathrm{e}-014$ \\
0.015 & 1.00955919930697 & $8.13209499739287 \mathrm{e}-011$ & $3.19744231092045 \mathrm{e}-014$ \\
0.020 & 1.01277612747026 & $1.34687372366216 \mathrm{e}-010$ & $8.12683254025615 \mathrm{e}-014$ \\
0.025 & 1.01600843431231 & $1.59590340942373 \mathrm{e}-010$ & $1.81854531433601 \mathrm{e}-013$ \\
0.030 & 1.01925618903530 & $2.13925543945948 \mathrm{e}-010$ & $4.32986979603811 \mathrm{e}-013$ \\
0.035 & 1.02251946118551 & $2.39566144699666 \mathrm{e}-010$ & $9.51905221313609 \mathrm{e}-013$ \\
0.040 & 1.02579832065467 & $2.94885227347663 \mathrm{e}-010$ & $1.99107397236276 \mathrm{e}-012$ \\
0.045 & 1.02909283768136 & $3.21276782955238 \mathrm{e}-010$ & $3.87823106962060 \mathrm{e}-012$ \\
0.050 & 1.03240308285239 & $3.77595510414608 \mathrm{e}-010$ & $7.14428516346288 \mathrm{e}-012$ \\
0.055 & 1.03572912710418 & $4.04751565596939 \mathrm{e}-010$ & $1.24744659046883 \mathrm{e}-011$ \\
0.060 & 1.03907104172416 & $4.62085258945422 \mathrm{e}-010$ & $2.08455475103619 \mathrm{e}-011$ \\
0.065 & 1.04242889835212 & $4.90019802512620 \mathrm{e}-010$ & $3.34869909579538 \mathrm{e}-011$ \\
0.070 & 1.04580276898164 & $5.48384226917165 \mathrm{e}-010$ & $5.20259391123545 \mathrm{e}-011$ \\
0.075 & 1.04919272596142 & $5.77111247679341 \mathrm{e}-010$ & $7.84587950164450 \mathrm{e}-011$ \\
0.080 & 1.05259884199672 & $6.36522168306897 \mathrm{e}-010$ & $1.15309317649803 \mathrm{e}-010$ \\
0.085 & 1.05602119015068 & $6.66056321207975 \mathrm{e}-010$ & $1.65603530888347 \mathrm{e}-010$ \\
0.090 & 1.05945984384577 & $7.26529947314702 \mathrm{e}-010$ & $2.33042252162363 \mathrm{e}-010$ \\
0.095 & 1.06291487686511 & $7.56885887298608 \mathrm{e}-010$ & $3.21983550932714 \mathrm{e}-010$ \\
0.100 & 1.06638636335390 & $7.64549534792991 \mathrm{e}-010$ & $4.37620162330177 \mathrm{e}-010$ \\
\hline
\end{tabular}


Table 3: The effector cells $x(t)$ obtained by DTM, the absolute error between $\chi_{R K 23}(t)$ and $x_{R K 45}(t)$, and the absolute error between $x(t)$ and $x_{R K 45}(t)$ at $x(0)=0.1$ and $y(0)=25$.

\begin{tabular}{|c|c|c|c|}
\hline $\mathrm{t}$ & $\chi(\mathrm{t})$ with DTM & $\left|\chi_{R K 23}(\mathrm{t})-\chi_{R K 45}(\mathrm{t})\right|$ & $\left|\chi(t)-\chi_{R K 45}(\mathrm{t})\right|$ \\
\hline 0 & 0.1 & 0 & 0 \\
0.005 & 0.100677982869877 & $1.29016242134128 \mathrm{e}-012$ & $1.63480340376054 \mathrm{e}-013$ \\
0.010 & 0.101357294986787 & $8.56163751006278 \mathrm{e}-012$ & $3.23754911768503 \mathrm{e}-013$ \\
0.015 & 0.102037943845018 & $2.19785023514163 \mathrm{e}-011$ & $4.92592078238374 \mathrm{e}-013$ \\
0.020 & 0.102719936865975 & $2.93700203046754 \mathrm{e}-011$ & $6.56155685341275 \mathrm{e}-013$ \\
0.025 & 0.103403281397298 & $4.30545737950538 \mathrm{e}-011$ & $8.32930946437216 \mathrm{e}-013$ \\
0.030 & 0.104087984711978 & $5.05615271872983 \mathrm{e}-011$ & $1.01449404432685 \mathrm{e}-012$ \\
0.035 & 0.104774054007478 & $6.45090647566349 \mathrm{e}-011$ & $1.22765686505488 \mathrm{e}-012$ \\
0.040 & 0.105461496404846 & $7.21265686065564 \mathrm{e}-011$ & $1.47540313299999 \mathrm{e}-012$ \\
0.045 & 0.106150318947837 & $8.63321775179671 \mathrm{e}-011$ & $1.79960213397834 \mathrm{e}-012$ \\
0.050 & 0.106840528602025 & $9.40551525552280 \mathrm{e}-011$ & $2.22172280572863 \mathrm{e}-012$ \\
0.055 & 0.107532132253927 & $1.08513614760497 \mathrm{e}-010$ & $2.80551970543996 \mathrm{e}-012$ \\
0.060 & 0.108225136710116 & $1.16336745792367 \mathrm{e}-010$ & $3.59766383351001 \mathrm{e}-012$ \\
0.065 & 0.108919548696339 & $1.31042607320886 \mathrm{e}-010$ & $4.68981797840939 \mathrm{e}-012$ \\
0.070 & 0.109615374856635 & $1.38960398743393 \mathrm{e}-010$ & $6.15896222910806 \mathrm{e}-012$ \\
0.075 & 0.110312621752454 & $1.53907886435434 \mathrm{e}-010$ & $8.12848399700528 \mathrm{e}-012$ \\
0.080 & 0.111011295861773 & $1.61914592844425 \mathrm{e}-010$ & $1.07078790279047 \mathrm{e}-011$ \\
0.085 & 0.111711403578212 & $1.77097725373443 \mathrm{e}-010$ & $1.40525785452539 \mathrm{e}-011$ \\
0.090 & 0.112412951210155 & $1.85187407075738 \mathrm{e}-010$ & $1.83027898392751 \mathrm{e}-011$ \\
0.095 & 0.113115944979865 & $2.00599911681643 \mathrm{e}-010$ & $2.36416858312438 \mathrm{e}-011$ \\
0.100 & 0.113820391022602 & $2.03356179118153 \mathrm{e}-010$ & $3.02366326421222 \mathrm{e}-011$ \\
\hline
\end{tabular}

Table 4: The tumor cells $y(t)$ obtained by DTM, the absolute error between $y_{R K 23}(t)$ and $y_{R K 45}(t)$, and the absolute error between $y(t)$ and $y_{R K 45}(t)$ at $x(0)=0.1$ and $y(0)=25$.

\begin{tabular}{|c|c|c|c|}
\hline $\mathrm{t}$ & $\mathrm{y}(\mathrm{t})$ with DTM & $\left|\mathrm{y}_{R K 23}(\mathrm{t})-\mathrm{y}_{R K 45}(\mathrm{t})\right|$ & $\left|\mathrm{y}(\mathrm{t})-\mathrm{y}_{R K 45}(\mathrm{t})\right|$ \\
\hline 0 & 25 & 0 & 0 \\
0.005 & 25.1823572542880 & $2.20131823880365 \mathrm{e}-009$ & $9.94759830064140 \mathrm{e}-014$ \\
0.010 & 25.3658826845262 & $1.97772962451381 \mathrm{e}-008$ & $5.75184344597801 \mathrm{e}-012$ \\
0.015 & 25.5505817918929 & $3.74754236531771 \mathrm{e}-008$ & $4.32009983342141 \mathrm{e}-012$ \\
0.020 & 25.7364600774028 & $5.53115562240691 \mathrm{e}-008$ & $2.23039364755095 \mathrm{e}-011$ \\
0.025 & 25.9235230415087 & $7.33401606112238 \mathrm{e}-008$ & $1.11068487740340 \mathrm{e}-010$ \\
0.030 & 26.1117761837030 & $9.14354387759886 \mathrm{e}-008$ & $3.30182103880361 \mathrm{e}-010$ \\
0.035 & 26.3012250021188 & $1.09795269764845 \mathrm{e}-007$ & $8.51976267313148 \mathrm{e}-010$ \\
0.040 & 26.4918749931317 & $1.28148577971388 \mathrm{e}-007$ & $1.89817583873264 \mathrm{e}-009$ \\
0.045 & 26.6837316509610 & $1.46840246628699 \mathrm{e}-007$ & $3.86661014317724 \mathrm{e}-009$ \\
0.050 & 26.8768004672710 & $1.65450334321804 \mathrm{e}-007$ & $7.27842319747651 \mathrm{e}-009$ \\
0.055 & 27.0710869307728 & $1.84474345132912 \mathrm{e}-007$ & $1.29154820172062 \mathrm{e}-008$ \\
0.060 & 27.2665965268255 & $2.03339837412386 \mathrm{e}-007$ & $2.17783231448720 \mathrm{e}-008$ \\
0.065 & 27.4633347370375 & $2.22696570517655 \mathrm{e}-007$ & $3.52341942289058 \mathrm{e}-008$ \\
0.070 & 27.6613070388684 & $2.41815961032898 \mathrm{e}-007$ & $5.49857617215821 \mathrm{e}-008$ \\
0.075 & 27.8605189052297 & $2.61505672227713 \mathrm{e}-007$ & $8.32298461261871 \mathrm{e}-008$ \\
0.080 & 28.0609758040868 & $2.80877330283147 \mathrm{e}-007$ & $1.22636979682511 \mathrm{e}-007$ \\
0.085 & 28.2626831980604 & $3.00900143912486 \mathrm{e}-007$ & $1.76520774886058 \mathrm{e}-007$ \\
0.090 & 28.4656465440275 & $3.20522303809412 \mathrm{e}-007$ & $2.48828364135534 \mathrm{e}-007$ \\
0.095 & 28.6698712927233 & $3.40878223425989 \mathrm{e}-007$ & $3.44320458367520 \mathrm{e}-007$ \\
0.100 & 28.8753628883424 & $3.45386485633981 \mathrm{e}-007$ & $4.68578395640407 \mathrm{e}-007$ \\
\hline
\end{tabular}




\section{Residual functions and residual errors}

We consider $x(t)$ and $y(t)$ are the solutions of system (3.1) by using DTM. Then, $R_{1}(t)$ and $R_{2}(t)$ are residual functions which are defined as

$$
R_{1}(t)=\eta \frac{d x(t)}{d t}+y(t) \frac{d x(t)}{d t}-\eta \sigma-\sigma y(t)+\eta \delta x(t)-(\rho-\delta-\eta \mu) x(t) y(t)+\mu x(t) y(t)^{2}
$$

and

$$
R_{2}(t)=\frac{d y(t)}{d t}-r_{2} y(t)(1-\beta y(t))+x(t) y(t)
$$

5.1. First, tumor dormant case, at $x(0)=1$ and $y(0)=1$

The residual functions $R_{1}(t)$ and $R_{2}(t)$ can be defined as

$$
\begin{aligned}
\mathrm{R}_{1}(\mathrm{t})= & 1 * 10^{-9}+1 * 10^{-10} \mathrm{t}^{2}+2 * 10^{-11} \mathrm{t}^{3}-2 * 10^{-12} \mathrm{t}^{4}-0.310840407 * 10^{-3} \mathrm{t}^{5} \\
& +0.3401723959 * 10^{-3} \mathrm{t}^{6}-0.6357405369 * 10^{-4} \mathrm{t}^{7}+0.1243305477 * 10^{-4} \mathrm{t}^{8} \\
& -0.1068678460 * 10^{-5} \mathrm{t}^{9}+1.082793505 * 10^{-8} \mathrm{t}^{10}+2.470632109 * 10^{-8} \mathrm{t}^{11} \\
& +4.282416979 * 10^{-9} \mathrm{t}^{12}+4.443535294 * 10^{-10} \mathrm{t}^{13}+5.660507120 * 10^{-11} \mathrm{t}^{14}+2.018749629 * 10^{-12} \mathrm{t}^{15},
\end{aligned}
$$

and

$$
\begin{aligned}
\mathrm{R}_{2}(\mathrm{t})= & -2 * 10^{-10} \mathrm{t}+1 * 10^{-10} \mathrm{t}^{2}+2 * 10^{-11} \mathrm{t}^{4}-0.2632137324 * 10^{-2} \mathrm{t}^{5} \\
& +0.4203074608 * 10^{-3} \mathrm{t}^{6}+0.2185861797 * 10^{-3} \mathrm{t}^{7}+0.1472760884 * 10^{-4} \mathrm{t}^{8} \\
& +0.4404530911 * 10^{-5} \mathrm{t}^{9}+2.204951939 * 10^{-7} \mathrm{t}^{10} .
\end{aligned}
$$

We can put values of $t$ take numbers $0,0.005,0.01, \ldots, 0.1$ to calculate the residual errors of $x(t)$ and $y(t)$. Table 5 shows the absolute residual errors of $x(t)$ and $y(t)$ for system (3.1).

Table 5: The effector cells $x(t)$ with its absolute residual error $\left|R_{1}(t)\right|$ and the tumor cells $y(t)$ with its absolute residual error $\left|R_{2}(t)\right|$ at $x(0)=1$ and $y(0)=1$.

\begin{tabular}{|c|c|c|c|c|}
\hline $\mathrm{t}$ & $\mathrm{x}(\mathrm{t})$ & $\left|\mathrm{R}_{1}(\mathrm{t})\right|$ & $\mathrm{y}(\mathrm{t})$ & $\left|\mathrm{R}_{2}(\mathrm{t})\right|$ \\
\hline 0 & 1 & $1 \mathrm{e}-009$ & 1 & 0 \\
0.005 & 0.998971532568814 & $1.00000153643271 \mathrm{e}-009$ & 1.00317120392005 & $1.00571883225062 \mathrm{e}-012$ \\
0.010 & 0.997945486538256 & $9.99979275477198 \mathrm{e}-010$ & 1.00635758096296 & $2.25279103760024 \mathrm{e}-012$ \\
0.015 & 0.996921859809352 & $9.99790386910482 \mathrm{e}-010$ & 1.00955919930697 & $4.97145331782907 \mathrm{e}-012$ \\
0.020 & 0.995900650294504 & $9.99067160353888 \mathrm{e}-010$ & 1.01277612747026 & $1.23556563897146 \mathrm{e}-011$ \\
0.025 & 0.994881855917514 & $9.97109924638311 \mathrm{e}-010$ & 1.01600843431231 & $3.05380077077747 \mathrm{e}-011$ \\
0.030 & 0.993865474613600 & $9.92783719938256 \mathrm{e}-010$ & 1.01925618903530 & $6.95597264049180 \mathrm{e}-011$ \\
0.035 & 0.992851504329413 & $9.84418698322884 \mathrm{e}-010$ & 1.02251946118551 & $1.44335523550740 \mathrm{e}-010$ \\
0.040 & 0.991839943023054 & $9.69714228565094 \mathrm{e}-010$ & 1.02579832065467 & $2.75613320582690 \mathrm{e}-010$ \\
0.045 & 0.990830788664094 & $9.45646681241710 \mathrm{e}-010$ & 1.02909283768136 & $4.90928718517666 \mathrm{e}-010$ \\
0.050 & 0.989824039233593 & $9.08380870349111 \mathrm{e}-010$ & 1.03240308285239 & $8.25554130300757 \mathrm{e}-010$ \\
0.055 & 0.988819692724112 & $8.53185127849203 \mathrm{e}-010$ & 1.03572912710418 & $1.32344248839387 \mathrm{e}-009$ \\
0.060 & 0.987817747139737 & $7.74349987750381 \mathrm{e}-010$ & 1.03907104172416 & $2.03816543944972 \mathrm{e}-009$ \\
0.065 & 0.986818200496097 & $6.65110456517172 \mathrm{e}-010$ & 1.04242889835212 & $3.03384593765644 \mathrm{e}-009$ \\
0.070 & 0.985821050820375 & $5.17571846790516 \mathrm{e}-010$ & 1.04580276898164 & $4.38608514807428 \mathrm{e}-009$ \\
0.075 & 0.984826296151334 & $3.22639151588172 \mathrm{e}-010$ & 1.04919272596142 & $6.18288357101930 \mathrm{e}-009$ \\
0.080 & 0.983833934539331 & $6.99499363414984 \mathrm{e}-011$ & 1.05259884199672 & $8.52555629827880 \mathrm{e}-009$ \\
0.085 & 0.982843964046333 & $2.52189273689096 \mathrm{e}-010$ & 1.05602119015068 & $1.15296423116702 \mathrm{e}-008$ \\
0.090 & 0.981856382745940 & $6.56863132892492 \mathrm{e}-010$ & 1.05945984384577 & $1.53258077341787 \mathrm{e}-008$ \\
0.095 & 0.980871188723399 & $1.15860416376687 \mathrm{e}-009$ & 1.06291487686511 & $2.00607429436284 \mathrm{e}-008$ \\
0.100 & 0.979888380075622 & $1.77344601626558 \mathrm{e}-009$ & 1.06638636335390 & $2.58980534585612 \mathrm{e}-008$ \\
\hline
\end{tabular}


5.2. Second, tumor escape case, at $x(0)=0.1$ and $y(0)=25$

The residual functions $R_{1}(t)$ and $R_{2}(t)$ can be defined as

$$
\begin{aligned}
\mathrm{R}_{1}(\mathrm{t})= & 2 * 10^{-9}+2 * 10^{-9} \mathrm{t}-2 * 10^{-9} \mathrm{t}^{2}-3 * 10^{-9} \mathrm{t}^{3}-2 * 10^{-10} \mathrm{t}^{4}+0.123168892 * 10^{-1} \mathrm{t}^{5} \\
& -0.648978592 * 10^{-1} \mathrm{t}^{6}-0.5304232022 * 10^{-1} \mathrm{t}^{7}-0.968174017 * 10^{-2} \mathrm{t}^{8} \\
& -0.1437512134 * 10^{-1} \mathrm{t}^{9}-0.6055046260 * 10^{-2} \mathrm{t}^{10}+0.8543839527 * 10^{-3} \mathrm{t}^{11} \\
& +0.6771444150 * 10^{-3} \mathrm{t}^{12}+0.1510306446 * 10^{-3} \mathrm{t}^{13}-0.1599991276 * 10^{-4} \mathrm{t}^{14} \\
& -0.8268108326 * 10^{-5} \mathrm{t}^{15}
\end{aligned}
$$

and

$$
\begin{aligned}
\mathrm{R}_{2}(\mathrm{t})= & -1 * 10^{-8} \mathrm{t}+1 * 10^{-8} \mathrm{t}^{2}+6 * 10^{-10} \mathrm{t}^{3}+2.788892571 * \mathrm{t}^{5} \\
& -0.3347543408 * \mathrm{t}^{6}-0.2768737728 * \mathrm{t}^{7}-0.7924100804 * 10^{-1} \mathrm{t}^{8} \\
& +0.4645930203 * 10^{-2} \mathrm{t}^{9}+0.6197634292 * 10^{-2} \mathrm{t}^{10}
\end{aligned}
$$

We can put values of $t$ take numbers $0,0.005,0.01, \ldots, 0.1$ to calculate the residual errors of $x(t)$ and $y(t)$. Table 6 shows the absolute residual errors of $x(t)$ and $y(t)$ for system (3.1).

Table 6: The effector cells $x(t)$ with its absolute residual error $\left|R_{1}(t)\right|$ and the tumor cells $y(t)$ with its absolute residual error $\left|R_{2}(t)\right|$ at $x(0)=0.1$ and $y(0)=25$.

\begin{tabular}{|c|c|c|c|c|}
\hline $\mathrm{t}$ & $\chi(\mathrm{t})$ & $\left|\mathrm{R}_{1}(\mathrm{t})\right|$ & $\mathrm{y}(\mathrm{t})$ & $\left|\mathrm{R}_{2}(\mathrm{t})\right|$ \\
\hline 0 & 0.1 & $2 \mathrm{e}-009$ & 25 & 0 \\
0.005 & 0.100677982869877 & $2.00998709697696 \mathrm{e}-009$ & 25.1823572542880 & $4.10398879139079 \mathrm{e}-011$ \\
0.010 & 0.101357294986787 & $2.02096325765499 \mathrm{e}-009$ & 25.3658826845262 & $1.79552326102079 \mathrm{e}-010$ \\
0.015 & 0.102037943845018 & $2.03814468728677 \mathrm{e}-009$ & 25.5505817918929 & $1.96620675055632 \mathrm{e}-009$ \\
0.020 & 0.102719936865975 & $2.07436840100688 \mathrm{e}-009$ & 25.7364600774028 & $8.70668032483199 \mathrm{e}-009$ \\
0.025 & 0.103403281397298 & $2.15281568670383 \mathrm{e}-009$ & 25.9235230415087 & $2.69081092783847 \mathrm{e}-008$ \\
0.030 & 0.104087984711978 & $2.30894203196480 \mathrm{e}-009$ & 26.1117761837030 & $6.72289626365258 \mathrm{e}-008$ \\
0.035 & 0.104774054007478 & $2.59159202886009 \mathrm{e}-009$ & 26.3012250021188 & $1.45506783408506 \mathrm{e}-007$ \\
0.040 & 0.105461496404846 & $3.06327757532582 \mathrm{e}-009$ & 26.4918749931317 & $2.83781602860567 \mathrm{e}-007$ \\
0.045 & 0.106150318947837 & $3.79959748576078 \mathrm{e}-009$ & 26.6837316509610 & $5.11314912047870 \mathrm{e}-007$ \\
0.050 & 0.106840528602025 & $4.88777640596971 \mathrm{e}-009$ & 26.8768004672710 & $8.65604073554944 \mathrm{e}-007$ \\
0.055 & 0.107532132253927 & $6.42430069855861 \mathrm{e}-009$ & 27.0710869307728 & $1.39339205517475 \mathrm{e}-006$ \\
0.060 & 0.108225136710116 & $8.51162872411664 \mathrm{e}-009$ & 27.2665965268255 & $2.15167236604186 \mathrm{e}-006$ \\
0.065 & 0.108919548696339 & $1.12539526908052 \mathrm{e}-008$ & 27.4633347370375 & $3.20868907452584 \mathrm{e}-006$ \\
0.070 & 0.109615374856635 & $1.47519889801217 \mathrm{e}-008$ & 27.6613070388684 & $4.64493178598678 \mathrm{e}-006$ \\
0.075 & 0.110312621752454 & $1.90967735794205 \mathrm{e}-008$ & 27.8605189052297 & $6.55412545729649 \mathrm{e}-006$ \\
0.080 & 0.111011295861773 & $2.43624389620624 \mathrm{e}-008$ & 28.0609758040868 & $9.04421492383637 \mathrm{e}-006$ \\
0.085 & 0.111711403578212 & $3.05979484536423 \mathrm{e}-008$ & 28.2626831980604 & $1.22383440134961 \mathrm{e}-005$ \\
0.090 & 0.112412951210155 & $3.78177638074264 \mathrm{e}-008$ & 28.4656465440275 & $1.62758291210169 \mathrm{e}-005$ \\
0.095 & 0.113115944979865 & $4.59914213837227 \mathrm{e}-008$ & 28.6698712927233 & $2.13131271148501 \mathrm{e}-005$ \\
0.100 & 0.113820391022602 & $5.50319919862528 \mathrm{e}-008$ & 28.8753628883424 & $2.75247974475332 \mathrm{e}-005$ \\
\hline
\end{tabular}

\section{Numerical Solutions}

We solve Kuznetsov and Taylor's system by repeating DTM procedure in small interval with two different initial values: $x(0)=1, y(0)=1$ (dormant tumor) and $x(0)=0.1, y(0)=25$ (tumor escape). We compare the solutions of the system; the effector cells $x(t), x_{R K 23}(t)$, and $x_{R K 45}(t)$ and tumor cells $y(t)$, 
$y_{R K 23}(t)$, and $y_{R K 45}(t)$, by three numerical methods: DTM, the Runge-Kutta second-third order method (RK23), and the Runge-Kutta fourth-fifth order method (RK45) (as exact solution).

Let $x(t)=x(a, b, t)$ and $y(t)=y(a, b, t)$, where $x(0)=a$ and $y(0)=b$. By using maple, we find DTM's terms

$$
\begin{aligned}
& X_{0}(a, b)=a, \\
& X_{1}(a, b)=-1 * 10^{-5}\left(-2.384439 * 10^{7}-1.181 * 10^{6} b+7.5571170 * 10^{7} a-6.939091 * 10^{6} a b\right. \\
& \left.+31100 a b^{2}\right) /(2019+100 b) \text {, } \\
& \mathrm{X}_{2}(\mathrm{a}, \mathrm{b})=1 * 10^{-11}\left(2.855500868 * 10^{16} \mathrm{a}+3.810442014 * 10^{14} \mathrm{~b}+1.239796968 * 10^{16} \mathrm{ab}\right. \\
& +3.726752971 * 10^{13} b^{2}+1.262477971 * 10^{14} \mathrm{ab}^{2}-4.496585476 * 10^{12} \mathrm{ab}^{3} \\
& -1.078357086 * 10^{16} a^{2} b+6.27909 * 10^{13} a^{2} b^{2}+9.92401 * 10^{9} b^{4} a \\
& \left.+1.555 * 10^{12} a^{2} b^{3}-9.009742251 * 10^{15}-1.836455 * 10^{11} b^{3}\right) /(2019+100 b)^{2}, \\
& X_{3}(a, b)=-6.666666667 * 10^{-18}\left(1.078967708 * 10^{25} a-3.750031782 * 10^{24} b\right. \\
& -8.253336827 * 10^{24} \mathrm{ab}-1.759832763 * 10^{23} \mathrm{~b}^{2}+4.193185481 * 10^{23} \mathrm{ab}^{2} \\
& +1.526469283 * 10^{22} a b^{3}+1.932049905 * 10^{25} a^{2} b-6.943893226 * 10^{23} a^{2} b^{2} \\
& +4.095488478 * 10^{20} \mathrm{~b}^{4} \mathrm{a}-2.567902965 * 10^{22} \mathrm{a}^{2} \mathrm{~b}^{3}+8.843499467 * 10^{20} \mathrm{~b}^{3} \\
& +3.857158977 * 10^{19} b^{4}-1.088601479 * 10^{25} a^{3} b-3.271627149 * 10^{18} a b^{5} \\
& +3.849211297 * 10^{20} a^{2} b^{4}+6.659533703 * 10^{23} a^{3} b^{2}+4.7093175 * 10^{21} a^{3} b^{3} \\
& +4.790319627 * 10^{15} a b^{6}+1.730404 * 10^{18} a^{2} b^{5}+7.775 * 10^{19} a^{3} b^{4} \\
& \left.-3.404383817 * 10^{24}-8.864568285 * 10^{16} b^{5}\right) /(2019+100 b)^{3} \text {, } \\
& \mathrm{Y}_{0}(\mathrm{a}, \mathrm{b})=\mathrm{b} \text {, } \\
& Y_{1}(a, b)=1.636 b-0.3272 * 10^{-2} b^{2}-a b, \\
& \mathrm{Y}_{2}(\mathrm{a}, \mathrm{b})=6.4 * 10^{-11} \mathrm{~b}\left(4.035469941 * 10^{13}+1.745441621 * 10^{12} \mathrm{~b}-1.220833466 * 10^{10} \mathrm{~b}^{2}\right. \\
& -4.570668984 * 10^{13} a-2.943534422 * 10^{12} a b+1.67281 * 10^{7} b^{3} \\
& \left.+1.00984375 * 10^{10} \mathrm{ab}^{2}+1.57734375 * 10^{13} \mathrm{a}^{2}+7.8125 * 10^{11} \mathrm{a}^{2} \mathrm{~b}\right) /(2019+100 \mathrm{~b}), \\
& \mathrm{Y}_{3}(\mathrm{a}, \mathrm{b})=-6.666666667 * 10^{-25} \mathrm{~b}\left(6.092349883 * 10^{30} \mathrm{a}-3.207176583 * 10^{29} \mathrm{~b}\right. \\
& +8.44597066 * 10^{29} \mathrm{ab}-3.493190164 * 10^{27} \mathrm{~b}^{2}+1.991743093 * 10^{28} \mathrm{ab}^{2} \\
& -2.556783059 * 10^{26} a b^{3}-5.744360193 * 10^{29} a^{2} b-1.437727320 * 10^{28} a^{2} b^{2} \\
& +4.7255877 * 10^{23} b^{4} a+8.836 * 10^{25} a^{2} b^{3}-3.857358506 * 10^{30} a^{2} \\
& +1.274702351 * 10^{26} \mathrm{~b}^{3}-5.042320361 * 10^{23} \mathrm{~b}^{4}+1.0095 * 10^{29} \mathrm{a}^{3} \mathrm{~b} \\
& +2.5 * 10^{27} \mathrm{a}^{3} \mathrm{~b}^{2}+1.01909025 * 10^{30} \mathrm{a}^{3}+5.254496947 * 10^{20} \mathrm{~b}^{5}-3.916687378 * 10^{30} \text { ) } \\
& /(2019+100 b)^{2} \text {. }
\end{aligned}
$$

We get a good DTM's solutions by using presented method with only three iterations where $x(a, b, t)=$ $X_{0}(a, b)+X_{1}(a, b) * t+X_{2}(a, b) * t^{2}+X_{3}(a, b) * t^{3}$ and $y(a, b, t)=Y_{0}(a, b)+Y_{1}(a, b) * t+Y_{2}(a, b) * t^{2}+$ $Y_{3}(a, b) * t^{3}$.

By using MATLAB, we use DTM procedure $(x(a, b, t), y(a, b, t))$ in small interval $[0,0.025)$ and repeat DTM procedure with change initial conditions to new initial conditions. Let, in first time, we use that $a_{0}=x(a, b, 0)$ and $b_{0}=y(a, b, 0)$, in second time, we get $a_{1}=x\left(a_{0}, b_{0}, 0.025\right)$ and $b_{1}=y\left(a_{0}, b_{0}, 0.025\right)$, in third time, we have $a_{2}=x\left(a_{1}, b_{1}, 0.05\right)$ and $b_{2}=y\left(a_{1}, b_{1}, 0.05\right)$, and so on.

We can repeat the last procedure, to any time we need, until stability of solution (the suitable interval of solution). 
First, $x(0)=1, y(0)=1$ (dormant tumor), we use DTM procedure in small interval $[0,0.025)$ and repeat DTM procedure iteration until $t=50$, the suitable interval of solution $[0,50)$, and compare with RK45 and RK23. This is shown in Figures 2 (a), (b), and (c).

Second, $x(0)=0.1, y(0)=25$ (tumor escape), we use DTM procedure in small interval $[0,0.025)$ and repeat DTM procedure iteration until $t=25$, the suitable interval of solution $[0,25)$, and compare with RK45 and RK23. This is shown in Figures 3 (a), (b), and (c).

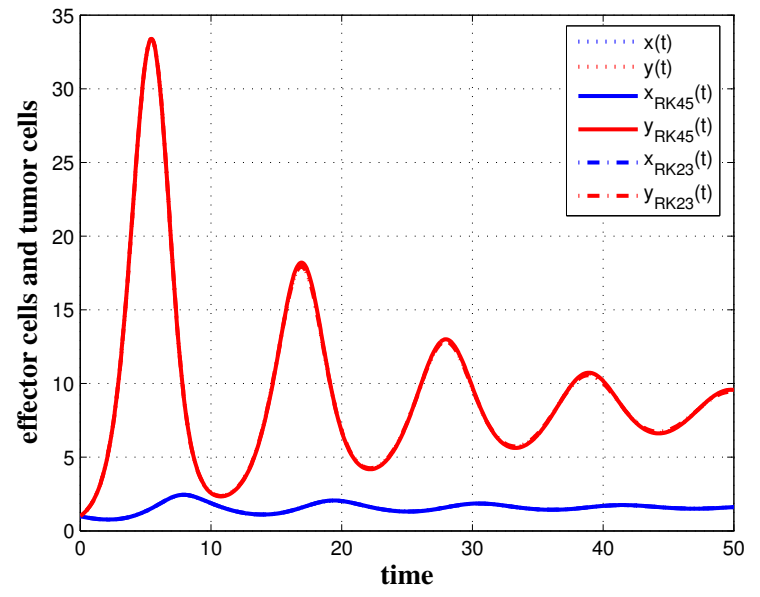

(a) (Tumor dormant case) at $x(0)=1$ and $y(0)=1$.

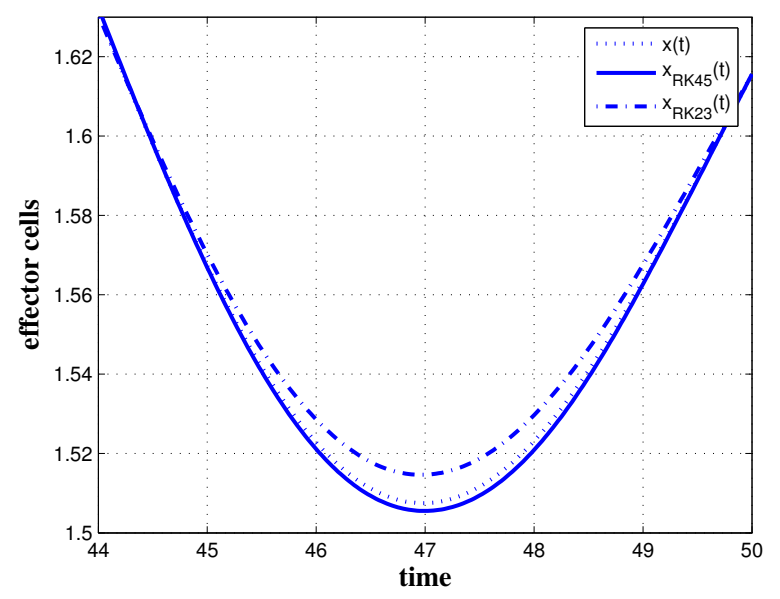

(b) (Effector cells) at $t=44$ to $t=50$.

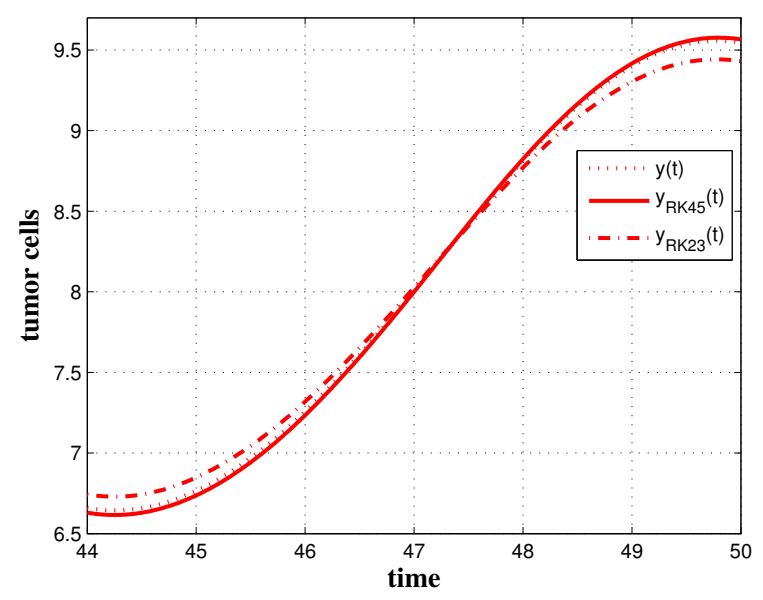

(c) (Tumor cells) at $\mathrm{t}=44$ to $\mathrm{t}=50$.

Figure 2: The effector cells with RK45 blue[-], the effector cells with RK23 blue[ -.-.- ], and the effector cells with DTM x(t) blue[ .... ] (lower line). The tumor cells with RK45 red[-], the tumor cells with RK23 red[ -.-.- ], and the tumor cells with DTM $\mathrm{y}(\mathrm{t}) \mathrm{red}[\ldots .$.$] (upper line).$

\section{Conclusion}

Differential transform method has been applied to non-linear system of ordinary differential equations, Kuznetsov and Taylor's system (tumor-immune system). There are two different cases in analysis of Kuznetsov and Taylor's system; tumor dormant case and tumor escape case. First, tumor dormant case, the immune system of animal body can resist and adobt cancer. Second, tumor escape case, cancer can destroy the immune system of animal body and increase without any resistance. We applied DTM 


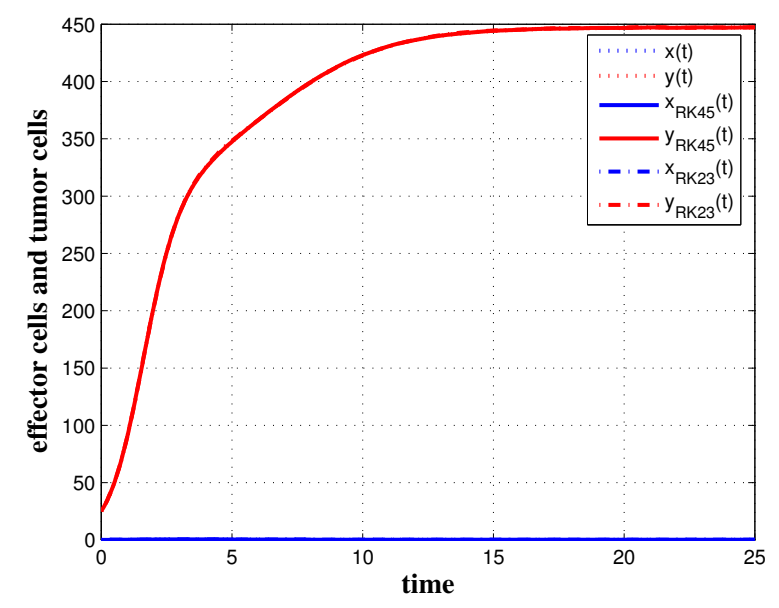

(a) (Tumor escape case) at $x(0)=0.1$ and $y(0)=25$.

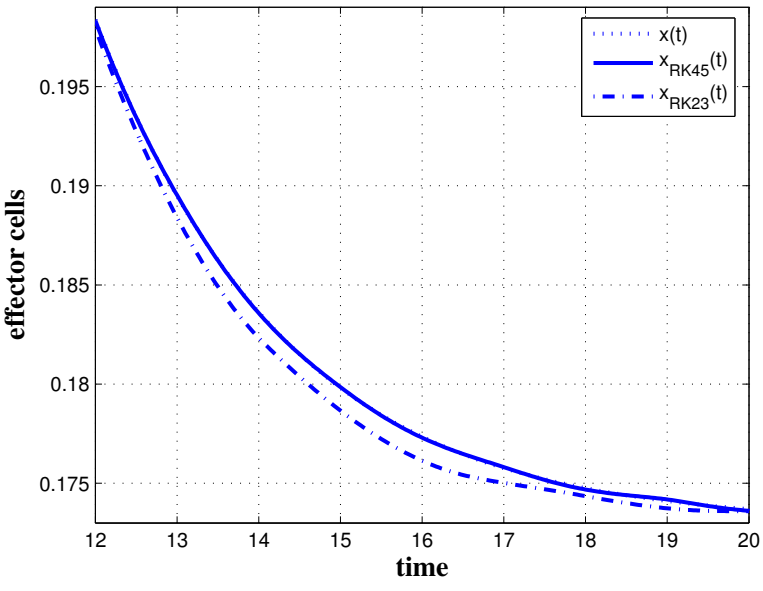

(b) (Effector cells) at $t=12$ to $t=20$.

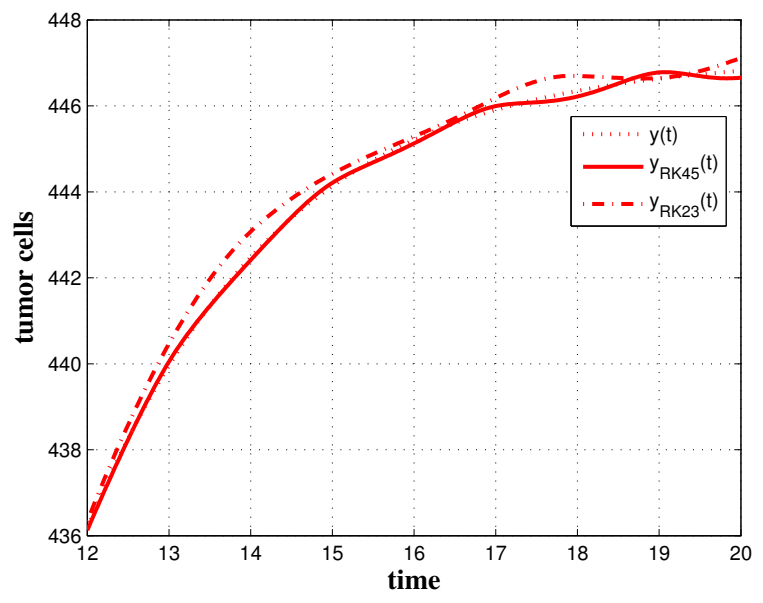

(c) (Tumor cells) at $t=12$ to $t=20$.

Figure 3: The effector cells with RK45 blue[-], the effector cells with RK23 blue[ -.-.- ], and the effector cells with DTM x(t) blue [ .... ] (lower line). The tumor cells with RK45 red[-], the tumor cells with RK23 red[ -...- ], and the tumor cells with DTM $\mathrm{y}(\mathrm{t}) \mathrm{red}[\ldots .$.$] (upper line).$

method on Kuznetsov and Taylor's system in two different cases; tumor dormant case and tumor escape case to test the effectiveness of DTM method for solving Non-linear system of ordinary differential equations at different initial conditions. In two cases, in small interval after start of initial conditions, the absolute error between DTM with only five iterations and RK45 is tiny (very small). Also, we find that the absolute error between DTM and RK45 is smaller than the absolute error between RK23 and RK45. Therefore, DTM with only five iterations is better than RK23 in small interval after start of initial conditions, i.e., $[0,0.1]$. See Tables $1,2,3$, and 4.

Then, we concluded the residual functions of Kuznetsov and Taylor's system and calculated the residual errors by using DTM. In two cases of tumor, tumor dormant case, and tumor escape case, the absolute residual errors of DTM's solutions approach to zero in small interval after start of initial conditions. Therefore, the solutions by using DTM are nearly equal the exact solutions in small interval after start of initial conditions. See Tables 5 and 6.

Also, by using maple, we conclude the two formulas for $x(a, b, t)$ (effector cells) and $y(a, b, t)$ (tumor cells) as function of initial conditions $\left(a=x_{0}\right.$ and $\left.b=y_{0}\right)$ and time $(t)$. In these formulas for $x(a, b, t)$ and $y(a, b, t)$, we suffice only three iterations which give us a good approximation. Then, by using MATLAB, 
we use DTM procedure $x(a, b, t), y(a, b, t)$ in small interval $[0,0.025)$ and repeat DTM procedure with change initial conditions to new initial conditions. Also, We can repeat this procedure until stability of solution (the suitable interval of solution). The numerical solutions showed that the suitable interval of solution is $[0,50)$ in tumor dormant case and is $[0,25)$ in tumor escape case. In two cases of tumor, in suitable interval, we clear DTM's solution is nearer to RK45 as exact solution than RK23. We conclude DTM with only three iteration which is better than RK23 in all cases, if we repeat DTM procedure in small interval, i.e., [0, 0.025). See Figures 2 (a), (b), and (c) and 3 (a), (b), and (c).

\section{References}

[1] J. A. Adam, Effects of vascularization on lymphocyte/tumor cell dynamics: Qualitative features, Math. Comput. Modelling, 23 (1996), 1-10. 1

[2] A. Arikoglu, I. Ozkol, Solution of boundary value problems for integro-differential equations by using transform method, Appl. Math. Comput., 168 (2005), 1145-1158. 2

[3] A. K. Bahşi, N. Şahin, M. Sezer, A numerical algorithm with residual error estimation for solution of high-order Pantograph-type functional differential equations using Fibonacci polynomials, New Trends Math. Sci., 3 (2015), 90102. 1

[4] A. K. Bahşi, S. Yalcinbas, Fibonacci collocation method with a residual error function to solve linear Volterra integro differential equations, New Trends Math. Sci., 4 (2016), 1-14. 1

[5] J. Biazar, E. Babolian, R. Islam, Solution of the system of ordinary differential equations by Adomian decomposition method, Appl. Math. Comput., 147 (2004), 713-719. 1

[6] J. Biazar, S. M. Shafiof, A Simple Algorithm for Calculating Adomian Polynomials, Int. J. Contemp. Math. Sci., 2 (2007), 975-982. 1

[7] J. C. Butcher, Numerical methods for ordinary differential equations, John Wiley \& Sons, Chichester, (2003). 2

[8] J. Damirchi, T. Rahimi Shamami, Differential transform method for a nonlinear system of differential equations arising in HIV infection of CD4+T cells, Int. J. Nonlinear Anal. Appl., 7 (2016), 269-277. 1

[9] C. DeLisi, A. Rescigno, Immune surveillance and neoplasia-I. A minimal mathematical model, Bull. Math. Biology, 39 (1977), 201-221. 1

[10] M. Galach, Dynamics of the tumor-immune system comptition-the effect of time delay, Int. J. Appl. Math. Comput. Sci., 13 (2003), 395-406. 1

[11] C. H. C. Hussin, A. Kılıçman, On the Solution of Fractional Order Nonlinear Boundary Value Problems By Using Differential Transformation Method, Eur. J. Pure Appl. Math., 4 (2011), 174-185. 1

[12] A. Kilıçman, O. Altun, On the solutions of some boundary value problems by using differential transformation method with convolution terms, Filomat, 26 (2012), 917-928. 1

[13] A. Kılıçman, O. Altun, On higher-orderboundaryvalueproblemsbyusing differential transformationmethodwith convolution terms, J. Franklin Inst., 351 (2014), 631-642. 1

[14] A. Kılıçman, O. Altun, Some Remarks on the Fractional Sumudu Transform and Applications, Appl. Math. Inf. Sci., 8 (2014), 2881-2888. 1

[15] D. Kirschner, J. C. Panetta, Modeling immunotherapy of the tumor-immune interaction, J. Math. Biology, 37 (1998), 235-252. 1

[16] V. A. Kuznetsov, I. A. Makalkin, M. A. Taylor, A. S. Perelson, Nonlinear dynamics of immunogenic tumors: Parameter estimation and global bifurcation analysis, Bull. Math. Biology, 56 (1994), 295-321. 1, 3

[17] F. Mirzaee, Differential Transform Method for Solving Linear and Nonlinear Systems of Ordinary Differential Equations, Appl. Math. Sci. (Ruse), 5 (2011), 3465-3472. 1, 2

[18] S. D. Moon, A. B. Bhosale, P. P. Gajbhiye, G. G. Lonare, Solution of Non-Linear Differential Equations by Using Differential Transform Method, Int. J. Math. Stat. Invention, 2 (2014), 78-82. 1

[19] Z. M. Odibat, Differential transform method for solving Volterra integral equation with separable kernels, Math. Comput. Modelling, 48 (2008), 1144-1149. 2

[20] N. Patil, A. Khambayat, Differential Transform Method For Ordinary Differential Equations, J. Comput. Math. Sci., 3 (2012), 330-337. 1

[21] F. A. Rihan, M. Safan, M. A. Abdeen, D. Abdel-Rahman, Qualitative and Computational Analysis of a Mathematical Model for the Tumor-Immune Interactions, J. Appl. Math., 2012 (2012), 19 pages. 1

[22] J. K. Zhou, Differential transformation and Application for electrical circuits, Huazhong University Press, Wuhan, (1986). 2 